\title{
Marked central nervous system pathology in CD59 knockout rats following passive transfer of Neuromyelitis optica immunoglobulin $\mathrm{G}$
}

\author{
Xiaoming Yao and Alan S. Verkman*
}

\begin{abstract}
Neuromyelitis optica spectrum disorders (herein called NMO) is an inflammatory demyelinating disease of the central nervous system in which pathogenesis involves complement-dependent cytotoxicity (CDC) produced by immunoglobulin G autoantibodies targeting aquaporin-4 (AQP4-lgG) on astrocytes. We reported evidence previously, using $\mathrm{CD}_{59} 9^{-/}$mice, that the membrane-associated complement inhibitor CD59 modulates CDC in NMO (Zhang and Verkman, J. Autoimmun. 53:67-77, 2014). Motivated by the observation that rats, unlike mice, have human-like complement activity, here we generated $\mathrm{CD}_{5} 9^{-1-}$ rats to investigate the role of CD59 in NMO and to create NMO pathology by passive transfer of AQP4-IgG under conditions in which minimal pathology is produced in normal rats. $\mathrm{CD}_{59^{-/-}}$rats generated by CRISPR/Cas9 technology showed no overt phenotype at baseline except for mild hemolysis.

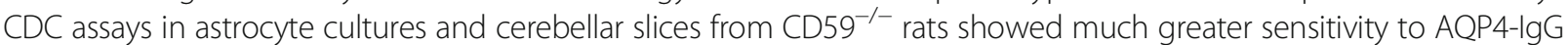
and complement than those from $\mathrm{CD}_{59^{+/+}}$rats. Intracerebral administration of AQP4-lgG in $\mathrm{CD} 59^{-/-}$rats produced marked NMO pathology, with astrocytopathy, inflammation, deposition of activated complement, and demyelination, whereas identically treated $\mathrm{CD} 59^{+/+}$rats showed minimal pathology. A single, intracisternal injection of AQP4-lgG in $\mathrm{CD}_{59^{-1}}$ rats produced hindlimb paralysis by 3 days, with inflammation and deposition of activated complement in spinal cord, optic nerves and brain periventricular and surface matter, with most marked astrocyte injury in cervical spinal cord. These results implicate an important role of CD59 in modulating NMO pathology in rats and demonstrate amplification of AQP4-IgG-induced NMO disease with CD59 knockout.
\end{abstract}

Keywords: NMO, Aquaporin-4, Complement inhibitor, Astrocyte, Complement-dependent cytotoxicity, Transgenic rat

\section{Introduction}

Neuromyelitis optica spectrum disorders (herein called $\mathrm{NMO}$ ) is an inflammatory demyelinating disease of the central nervous system with characteristic pathological features in spinal cord and optic nerves, and to a lesser extent in brain. Most NMO patients are seropositive for immunoglobulin G autoantibodies against astrocyte water channel aquaporin-4 (AQP4), called AQP4-IgG (or NMO-IgG) $[13,14]$. The major pathological features in seropositive NMO include astrocyte damage, inflammation with prominent granulocyte and macrophage

\footnotetext{
* Correspondence: Alan.Verkman@ucsf.edu; http://www.ucsf.edu/verklab Departments of Medicine and Physiology, University of California, San Francisco, CA, USA
}

infiltration, vasculocentric deposition of activated complement, and demyelination, which can produce marked neurological deficits $[10,19,26]$. There is abundant evidence that pathogenesis in AQP4-IgG seropositive NMO patients involves AQP4-IgG binding to AQP4 on astrocytes and activation of the classical complement system, which causes complement-dependent cytotoxicity (CDC) leading to inflammation, blood-brain barrier disruption and demyelination $[8,13,19]$. Antibodydependent cell-mediated cytotoxicity (ADCC) [24] and sensitized $\mathrm{T}$ cells $[22,35,36]$ may also play a role in NMO pathogenesis.

Several lines of evidence implicate a major role for complement activation in NMO, including human 
pathology showing deposition of activated complement $[16,18,26]$, rodent models showing complementdependent NMO pathology following passive transfer of AQP4-IgG [1, 28, 37], and an open-label clinical trial of the C5 convertase inhibitor eculizumab showing efficacy in NMO [21]. We previously reported that complement inhibitor protein CD59, a phosphoinositol-linked membrane glycoprotein expressed on astrocytes that inhibits formation of the terminal membrane attack complex, may be an important regulator of complement action in NMO [38]. CD59 ${ }^{-/-}$mice are highly sensitive to administration of AQP4-IgG and human complement, with longitudinally extensive NMO spinal cord pathology produced by coinjection of AQP4-IgG and complement into the lumbosacral cerebrospinal space. However, a major limitation of mice as models of NMO is the nearzero activity of their classical complement pathway, in part because of complement inhibitory factor(s) present in mouse serum [25]. The ineffective classical complement pathway in mice precludes the development of clinically relevant NMO models, such as robust passivetransfer models of NMO optic neuritis and transverse myelitis, as well as testing of NMO therapeutics such as complement inhibitors.

To overcome these limitations and to further investigate the role of CD59 in NMO pathogenesis, here we generated $\mathrm{CD} 59^{-/-}$rats and determined their sensitivity to passive transfer of AQP-IgG. We previously showed that passive transfer of AQP4-IgG to rats, without added complement, by a single intracerebral injection produced NMO pathology in brain at the site of injection [1]. We tested here the prediction that marked NMO pathology might be produced in the central nervous system by passive transfer of AQP4-IgG to $\mathrm{CD} 59^{-/-}$rats, without added complement, under conditions where minimal pathology is produced in $\mathrm{CD} 59^{+/+}$rats.

\section{Materials and methods $\mathrm{CD} 59^{-/-}$rats}

$\mathrm{CD} 59^{-1-}$ rats in a Sprague-Dawley background were custom-generated by Transposagen Biopharm. Inc. (Lexingtobon, KY) using CRISPR-Cas9 gene targeting technology. Exon 3 of the CD59 gene was targeted to induce sequence deletions with frame-shifts, which were identified by PCR genotyping and sequence analysis. Primers for PCR genotyping were: CD59-11 F (5' to $3^{\prime}$ : GGTCGA AGACATTTCTGGTTTAC) and CD59-11R (5' to $3^{\prime}$ : GACACAACAGCAGCCATTAC), followed by restriction enzyme digestion with $\mathrm{HpyCH} 4 \mathrm{~V}$ (Fig. 1a), which produced distinct bands corresponding to the wildtype and edited alleles (Fig. 1b). Breeding of $\mathrm{CD} 59^{+/-}$rats was done to generate wildtype $\left(\mathrm{CD} 59^{+/+}\right)$and $\mathrm{CD} 59^{-/-}$rats for experiments. In vivo studies were done on 8- to 10-week-old, weight-matched $\mathrm{CD} 59^{+/+}$and $\mathrm{CD} 59^{-/-}$rats. Rats were maintained in air-filtered cages and fed normal rat chow in the University of California, San Francisco (UCSF) Animal Care facility. All procedures were approved by the UCSF Committee on Animal Research.

\section{Materials}

Purified recombinant AQP4-IgG (rAb-53) was provided by Dr. Jeffrey Bennett (Univ. Colorado, Denver). Human complement was purchased from Innovative Research (Novi, MI) and human control IgG from Pierce Biotechnology (Rockford, IL). Unless otherwise specified chemicals were purchased from Sigma-Aldrich (St. Louis, MO).

\section{Astrocyte cell culture}

Primary astrocyte cultures were generated from brain cortex of neonatal $\mathrm{CD} 59^{+/+}$and $\mathrm{CD} 59^{-/-}$rats at day 7 post birth, as described [15] with modification. Briefly, the cerebral hemispheres were isolated and cortical tissue was minced and incubated for $15 \mathrm{~min}$ at $37{ }^{\circ} \mathrm{C}$ in $0.25 \%$ trypsin-EDTA. Dissociated cells were centrifuged and resuspended in Dulbecco's Modified Eagle's Medium (DMEM) containing 10\% FBS and 1\% penicillin/streptomycin, and grown at $37{ }^{\circ} \mathrm{C}$ in a $5 \% \mathrm{CO}_{2}$ incubator. After cell confluence (8-10 days), flasks were shaken in a rotator at $180 \mathrm{rpm}$ overnight to purify astrocytes and medium was replaced with DMEM containing 3\% FBS and $0.25 \mathrm{mM}$ dibutyryl cAMP. Cultures were maintained for an additional 2 weeks. Cultures contained $>95 \%$ astrocytes as shown by positive glial fibrillary acidic protein (GFAP) immunofluorescence.

\section{Complement-dependent cytotoxicity (CDC)}

Astrocyte cultures were trypsinized and plated onto 96well microplates at 20,000 cells/well and grown for $48 \mathrm{~h}$. Human complement and AQP4-IgG were added in Hank's balanced salt solution (HBSS, pH 7.2; Invitrogen), and cells were incubated at $28{ }^{\circ} \mathrm{C}$ for $2 \mathrm{~h}$ for cytotoxicity measurement by the Alamar Blue assay (Invitrogen) as described [38].

\section{Organotypic cerebellar slice cultures and ex vivo NMO model}

Cerebellar slice cultures were prepared using an interfaceculture method as described [34] with modification. Postnatal day $7 \mathrm{CD} 59^{+/+}$and $\mathrm{CD} 59^{-/-}$rats were decapitated and the whole cerebellum was removed, placed in ice-cold HBSS and embedded in $2 \%$ low-melting point agarose. Parasagittal slices of $300-\mu \mathrm{m}$ thickness were cut using a vibrating microtome and placed on transparent, non-coated membrane inserts (Millipore, Millicell-CM $0.4-\mu \mathrm{m}$ pores, $30-\mathrm{mm}$ diameter) in six-well plates containing $1 \mathrm{~mL}$ culture medium (50\% MEM, 25\% HBSS, 25\% horse serum, 1\% penicillin-streptomycin, $0.65 \%$ glucose and $25 \mathrm{mM}$ HEPES), with a thin film 


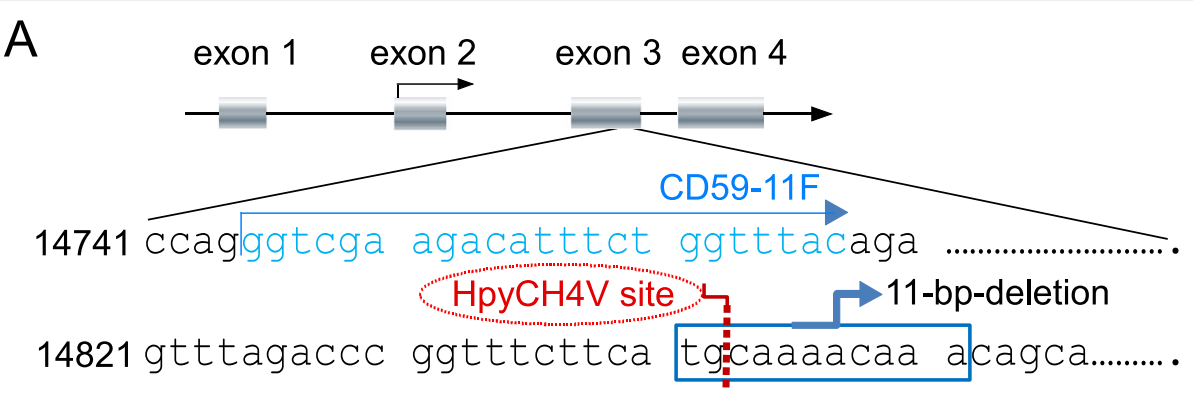

15001 agcagtaatg gctgctgttg tgtclatatt tegcettac

B

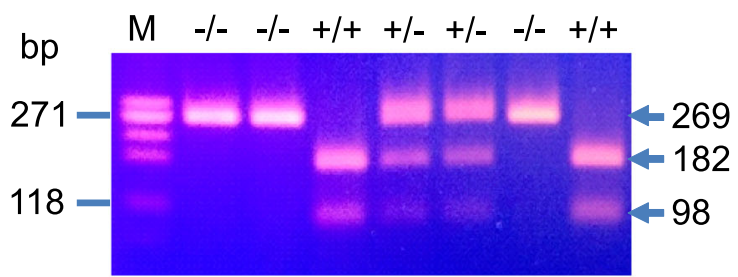

C

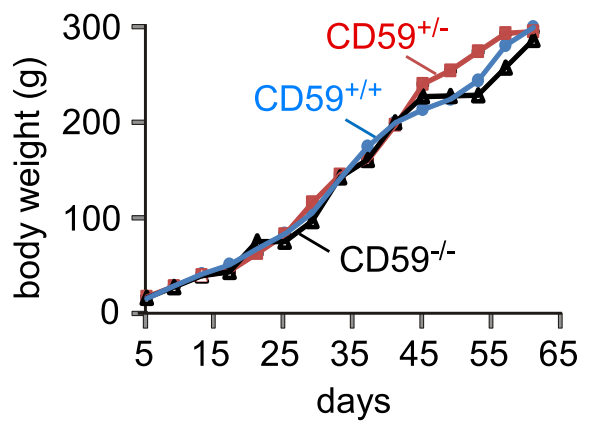

D

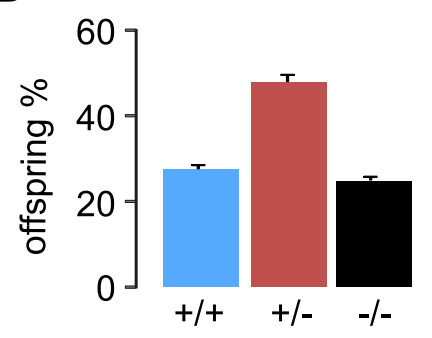

E
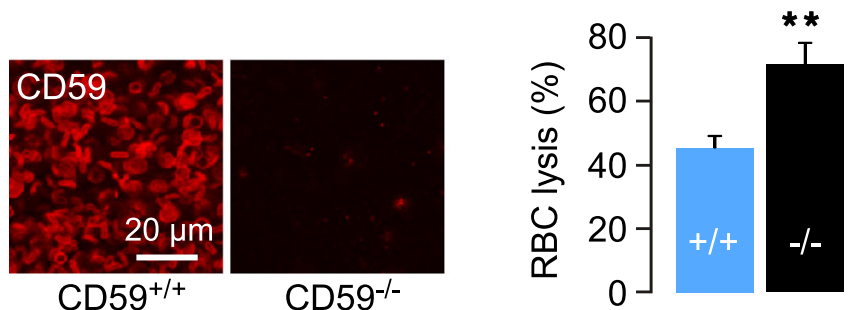

Fig. 1 Generation and characterization of $\mathrm{CD} 59^{-1-}$ rats. a. Schematic of CD59 gene deletion strategy showing deletion of 11 bp in exon 3, and PCR genotyping strategy. b. PCR genotype analysis following HpyCH4V restriction enzyme digestion, showing a single band at 269 bp for

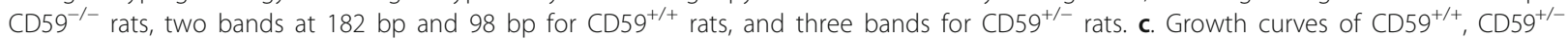
and $\mathrm{CD} 59^{-/-}$rats (20 rats per group, differences not significant). $\mathbf{d}$. Genotype distribution of 150 pups from breeding of CD59 ${ }^{+/-}$rats. e. (left) CD59 immunofluorescence in blood smears. (right) Percentage erythrocyte lysis following complement activation (mean \pm S.E.M., $n=6,{ }^{* *} P<0.01$ )

of culture medium covering slices. Slices were cultured in $5 \% \mathrm{CO}_{2}$ at $37{ }^{\circ} \mathrm{C}$ for 7 days with medium change every 2 days. AQP4-IgG (or control human IgG) and human complement were added on day 7 and slices were fixed $24 \mathrm{~h}$ later in $4 \%$ paraformaldehyde (PFA) for whole-mount immunostaining.

\section{Blood analysis}

Blood $(200 \mu \mathrm{L})$ was collected into EDTA tubes for cell analysis and into tubes without anticoagulant for serum. To study complement-mediated erythrocyte lysis, $100 \mu \mathrm{L}$ of fresh rat serum was placed in wells of a 96well plate and acidified by addition of $10 \mu \mathrm{L}$ of $0.2 \mathrm{~N}$ 
$\mathrm{HCl}$ to each well to give a $\mathrm{pH}$ of $6.5-6.8$, as described [23]. Erythrocytes $(10 \mu \mathrm{L}$ of $50 \%$ suspension in PBS) were added to each well, and hemolysis quantified by absorbance at $412 \mathrm{~nm}$ after $1 \mathrm{~h}$ incubation at $37{ }^{\circ} \mathrm{C}$, referenced against zero and $100 \%$ lysis controls. Hematology parameters were measured using a Genesis Hematology Analyzer (Oxford Science, Oxford, CT).

\section{Intracerebral injection model}

AQP4-IgG $(30 \mu \mathrm{g})$ was delivered by intracerebral injection as described [1] with modification. $\mathrm{CD} 59^{+/+}$and $\mathrm{CD}_{59^{-/-}}$rats were anesthetized with ketamine $(100 \mathrm{mg} /$ $\mathrm{kg})$ and xylazine $(10 \mathrm{mg} / \mathrm{kg})$ and mounted on a stereotaxic frame. A midline scalp incision was made and a burr hole of $1-\mathrm{mm}$ diameter was drilled on each side of the skull $0.5 \mathrm{~mm}$ anterior and $3.5 \mathrm{~mm}$ lateral to the bregma. A glass pipette with $40-\mu \mathrm{m}$ diameter tip was inserted at a depth of $5 \mathrm{~mm}$ to infuse AQP4-IgG (or control IgG) in a total volume of $3 \mu \mathrm{L}$ over $10 \mathrm{~min}$ by pressure injection. After injection, the glass pipette was kept in place for $10 \mathrm{~min}$ before slow withdrawal (over $5 \mathrm{~min}$ ) to prevent leaking. At day 7 rats were deeply anesthetized and transcardially perfused with $200 \mathrm{~mL}$ heparinized PBS and $200 \mathrm{~mL}$ of $4 \%$ PFA in PBS. Brains were removed and post-fixed for $4 \mathrm{~h}$ in $4 \%$ PFA and crytoprotected in $20 \%$ sucrose. Serial frozen coronal sections (thickness $7 \mu \mathrm{m}$ ) were cut on a cryostat.

\section{Intracisternal injection model}

AQP4-IgG was delivered by injection into the cisterna magna of $\mathrm{CD} 59^{+/+}$and $\mathrm{CD} 59^{-/-}$rats. Rats were anesthetized as above, mounted on a stereotaxic frame, the cisterna magna was exposed, and a glass pipette with 40$\mu \mathrm{m}$ tip diameter was inserted. AQP4-IgG or control IgG (15 or $30 \mu \mathrm{g}$ in $10 \mu \mathrm{L}$ artificial cerebrospinal fluid, aCSF) was infused at $2 \mu \mathrm{l} / \mathrm{min}$ over $5 \mathrm{~min}$ by pressure injection at 10 psi. In some experiments a recombinant monoclonal anti-AQP4 'aquaporumab' lacking effector functions (AQP4-IgG ${ }^{-C D C}$ ) [30] was infused. After injection, the glass pipette was withdrawn with no leakage seen. At specified times, rats were euthanized as above, and brain, spinal cord and optic nerves were removed for sectioning. Rat motor function was scored as described [20] with modification: score $0=$ normal movement; score 1, tail paralysis; score 2, hindlimb paralysis; score 3, hindlimb paralysis with frontlimb paresis; score 4, complete paralysis with moribund condition.

\section{Immunofluorescence}

Cultured astrocytes and cerebellar slice cultures were fixed with 4\% PFA for 15 min and incubated in blocking solution as described [34]. Frozen sections of brain, spinal cord and other organs were post-fixed with $4 \%$ PFA for $5 \mathrm{~min}$ and incubated in blocking solution as described [34]. Slides were then incubated for $2 \mathrm{~h}$ with antibodies against GFAP (1:200; Millipore), AQP4 (1:200, Santa Cruz Biotechnology), ionized calcium binding adaptor molecule 1 (Iba1; 1:400, Wako, Richmond, VA), CD45 (1:20, Cambridge, MA), C9neo (1:100, Santa Cruz Biotechnology), myelin basic protein (MBP, 1:100, Santa Cruz Biotechnology), human IgG (1:100, Santa Cruz Biotechnology), or CD59 (7A6, $5 \mu \mathrm{g} / \mathrm{mL}$, LSBio, Seattle, WA), followed by the appropriate species-specific Alexa Fluorconjugated secondary antibody for $1 \mathrm{~h}(5 \mu \mathrm{g} / \mathrm{mL}$ each, Invitrogen). Sections were mounted with VectaShield (Vector Laboratories, Burlingame, CA) for visualization of immunofluorescence on a Leica fluorescence microscope or Nikon confocal microscope.

\section{Statistics}

Data are presented as mean \pm S.E.M. Statistical analysis was performed using Prism 5 GraphPad Software package (San Diego, CA). The normality of the data was established by Bartlett's test for equal variances and a one-way ANOVA with Newmann-Keuls post-hoc test to compare groups.

\section{Results}

Generation and characterization of $\mathrm{CD} 59^{-/-}$rats

$\mathrm{CD} 59^{-1-}$ rats were generated as diagrammed in Fig. 1a. Following breeding and attempted expansion of 3 rat clones, we selected a clone that thrived well, which contained an 11-bp deletion in exon 3 of the rat CD59 gene. Genomic PCR analysis following restriction enzyme digestion of PCR products showed $\mathrm{CD} 59^{+/+}, \mathrm{CD} 59^{+/-}$and $\mathrm{CD} 59^{-/-}$rats from breeding of $\mathrm{CD} 59^{+/-}$rats (Fig. 1b). $\mathrm{CD} 59^{-/-}$rats showed no overt phenotype, including neurological function and behavior, with similar growth found for $\mathrm{CD}_{5} 9^{-/-}$and $\mathrm{CD} 59^{+/+}$rats (Fig. 1C), and breeding of $\mathrm{CD} 59^{+/-}$rats gave an approximate 1:2:1 distribution of viable $\mathrm{CD} 59^{+/-}, \mathrm{CD} 59^{+/-}$and $\mathrm{CD} 59^{-1-}$ offspring (Fig. 1d). CD59 immunofluorescence in multiple tissues (blood smear shown in Fig. 1e, left) confirmed undetectable CD59 protein in the $\mathrm{CD} 59^{-/-}$rats. Functional studies of complement-mediated erythrocyte lysis confirmed the expected greater lysis in $\mathrm{CD} 59^{-/-}$compared to $\mathrm{CD}_{59^{+/+}}$erythrocytes (Fig. 1e, right). Hematological parameters suggested a mild hemolytic anemia in $\mathrm{CD} 59^{-/-}$ rats as evidenced by reduced hematocrit and mild reticulocytosis (Table 1), as has been reported in humans lacking CD59 [12]. Peripheral tissues did not show inflammation, deposition of activated complement (C5b-9 immunofluorescence), or histological abnormalities (see below, and data not shown).

We note an interesting observation made in carrying out control studies (of AQP4-IgG administration to $\mathrm{CD} 59^{-/-}$rats) in which complement was inactivated by administration of cobra venom factor (350 units $/ \mathrm{kg}$ ), as 
Table 1 Hematological parameters in $\mathrm{CD}_{59^{-/-}}$and $\mathrm{CD}_{5} 9^{+/+}$rats

\begin{tabular}{llllllll}
\hline & $\mathrm{Hb}(\mathrm{g} / \mathrm{dl})$ & $\mathrm{RBC}\left(10^{12} / \mathrm{L}\right)$ & $\mathrm{HCT}(\%)$ & $\mathrm{RDW}(\%)$ & Retic $(\%)$ & WBC $\left(10^{9} / \mathrm{L}\right)$ & $\mathrm{Plt}\left(10^{9} / \mathrm{L}\right)$ \\
\hline $\mathrm{CD} 59^{+/+}$ & $14.8 \pm 0.5$ & $8.7 \pm 0.4$ & $40.3 \pm 1.5$ & $13.3 \pm 2.3$ & $2 \pm 0.1$ & $7.5 \pm 2.5$ & $737 \pm 165$ \\
$\mathrm{CD} 59^{-/-}$ & $14.3 \pm 0.2^{*}$ & $7.8 \pm 0.2^{*}$ & $38.5 \pm 1.2^{*}$ & $14.2 \pm 2^{*}$ & $12 \pm 0.1^{*}$ & $7.4 \pm 3.1$ & $729 \pm 188$
\end{tabular}

Abbreviations: $H b$ hemoglobin, $R B C$ red blood cell count, $H C T$ hematocrit, RDW RBC distribution width, Retic reticulocyte count, WBC white blood cell count, Plts platelet count

Mean \pm S.D. of 6 rats per genotype (three males and three females)

${ }^{*} P<0.01$ comparing $\mathrm{CD} 59^{-/-}$with $\mathrm{CD} 59^{+/+}$

we have done previously in $\mathrm{CD} 59^{+/+}$rats $[1,7]$. All $\mathrm{CD} 59^{-1-}$ rats receiving cobra venom factor became moribund and died within $12-24 \mathrm{~h}$, whereas no abnormalities were seen in $\mathrm{CD} 59^{+/+}$rats treated identically.

Immunofluorescence of CD59 and AQP4 in CD59/+ rats showed their gross coexpression in brain, spinal cord and optic nerve (Fig. 2a-c), in agreement with prior results [38]. We did not carry out high-resolution analysis of their cellular or subcellular localization. CD59 immunofluorescence of two major peripheral tissues in which AQP4 is expressed, kidney and skeletal muscle, also showed CD59 and AQP4 coexpression (Fig. 2d). CD59 immunofluorescence was not seen in CNS or peripheral tissues from $\mathrm{CD} 59^{-/-}$rats, and AQP4 immunofluorescence was similar in tissues from $\mathrm{CD} 59^{+/+}$ and $\mathrm{CD} 59^{-/-}$rats.

Marked complement-mediated injury in astrocyte cultures and brain slices from $\mathrm{CD} 59^{-/-}$rats

Complement-dependent cytotoxicity (CDC) was measured in primary astrocyte cultures generated from neonatal $\mathrm{CD}_{59^{+/+}}$and $\mathrm{CD} 59^{-/-}$rats. Immunofluorescence of astrocytes cultures from $\mathrm{CD} 59^{+/+}$rats showed CD59 coexpression with AQP4; similar AQP4 expression but without $\mathrm{CD} 59$ was seen on astrocytes from $\mathrm{CD} 59^{-/-}$rats (Fig. 3a). CDC was measured following 2-h incubation of astrocyte cultures with different concentrations of AQP4-IgG in the presence of human complement (Fig. 3b). $\mathrm{CD} 59^{-1-}$ astrocyte cultures were significantly more sensitive to AQP4-IgG-induced CDC than were $\mathrm{CD} 59^{+/+}$astrocyte cultures, similar to prior results in $\mathrm{CD} 59^{+/+}$and $\mathrm{CD} 59^{-/-}$mouse astrocyte cultures [38].

To confirm the predicted greater sensitivity of a CD $59^{-/-}$ CNS tissue to development of complement-mediated NMO-like pathology, ex vivo cultured cerebellar slices from $\mathrm{CD} 59^{+/+}$and $\mathrm{CD} 59^{-/-}$rats were incubated with AQP4-IgG and complement for 1 day. $\mathrm{CD} 59^{-/-}$cerebellar slices showed astrocyte injury with loss of AQP4 and GFAP immunofluorescence, seen most prominently at the peripheral border, and deposition of activated complement as seen by C5b-9 immunofluorescence (Fig. 3c). In contract, minimal loss of AQP4 and GFAP, and complement deposition were seen in $\mathrm{CD} 59^{+/+}$cerebellar slices under the same experimental conditions.

\section{Marked NMO pathology in brains of $\mathrm{CD} 59^{-/-}$rats following intracerebral AQP4-IgG injection}

Direct intracerebral injection of AQP4-IgG (without added complement) in rat brain has been shown to produce NMO-like pathology around the injection site, with loss of AQP4 and GFAP, deposition of activated complement, inflammation and demyelination [1]. To compare the sensitivity of $\mathrm{CD} 59^{+/+}$and $\mathrm{CD} 59^{-/-}$rats in this model, a submaximal amount of AQP4-IgG was injected into brain striatum (Fig. 4a). Under conditions in which minimal pathology was seen in $\mathrm{CD} 59^{+/+}$rats, there was marked astrocyte injury in the ipsilateral hemispheres of $\mathrm{CD} 59^{-1-}$ rats as seen by loss of AQP4 and GFAP immunofluorescence and demyelination as seen by loss of MBP immunofluorescence (Fig. 4b), as well as microglia activation (Iba-1 immunofluorescence), leukocyte infiltration (CD45 immunofluorescence), and deposition of activated complement (C5b-9 immunofluorescence). Figure 4c summarizes areas of loss of AQP4, GFAP and MBP immunofluorescence in the ipsilateral, AQP4-IgG injected hemisphere and the contralateral, control IgG injected hemisphere. We conclude that $\mathrm{CD} 59^{-/-}$rat brain is highly susceptible to development of NMO pathology following exposure to AQP4-IgG.

\section{NMO pathology in CNS tissues of $\mathrm{CD} 59^{-/-}$rats following intracisternal AQP4-lgG injection}

We next introduced AQP4-IgG into the CSF by intracisternal injection (Fig. 5a). In an initial study, injection of $30 \mu \mathrm{g} \mathrm{AQP4} 4-\mathrm{IgG}$ into the CSF of CD59 ${ }^{-/-}$rats produced marked paralysis by day 1 and death soon thereafter. In subsequent studies, intracisternal injection of a reduced, $15 \mu \mathrm{g}$ amount of AQP4-IgG produced motor dysfunction in all $\mathrm{CD} 59^{-/-}$rats by day 1 , but with $>80 \%$ survival on day 3; no hindlimb motor dysfunction or mortality was seen in $\mathrm{CD}_{5} 9^{+/+}$rats administered $15 \mu \mathrm{g}$ AQP4-IgG or in $\mathrm{CD} 59^{+/+}$or $\mathrm{CD} 59^{-/-}$rats administered $15 \mu \mathrm{g}$ control IgG or an engineered AQP4-IgG lacking complement effector function (Fig. 5b). Examination of skeletal muscle and kidney from AQP4-IgG treated CD59 $9^{-1-}$ rats did not show C5b-9 deposition or inflammation, and human IgG was undetectable in the serum (data not shown), suggesting that the gross motor dysfunction in the antibody-treated $\mathrm{CD} 59^{-/-}$rats is not a consequence of peripheral organ injury. 


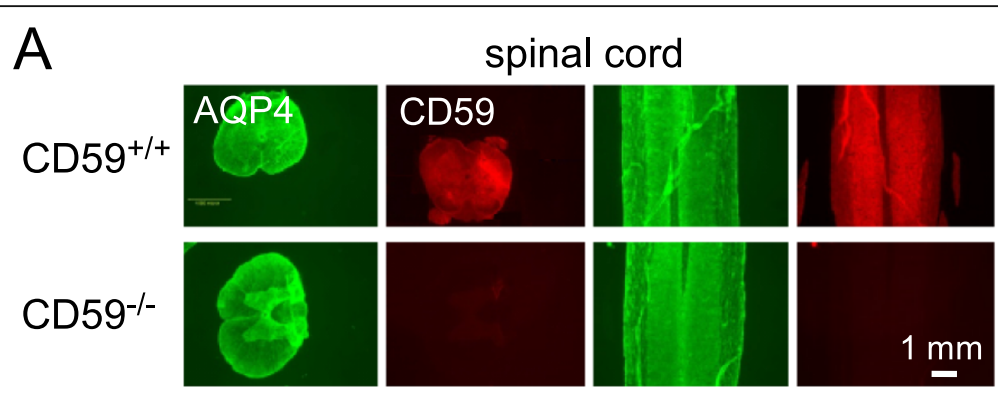

B

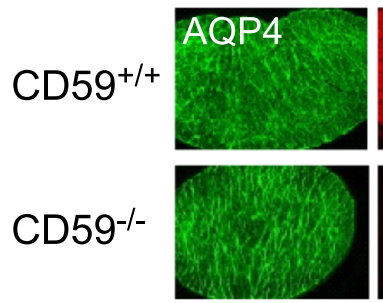

optic nerves
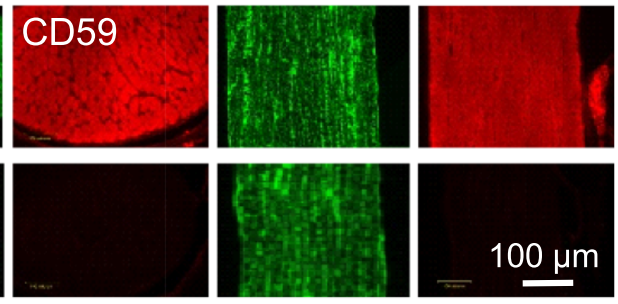

C
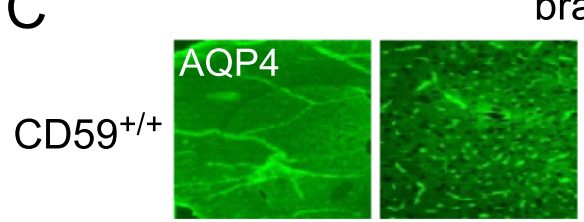

brain
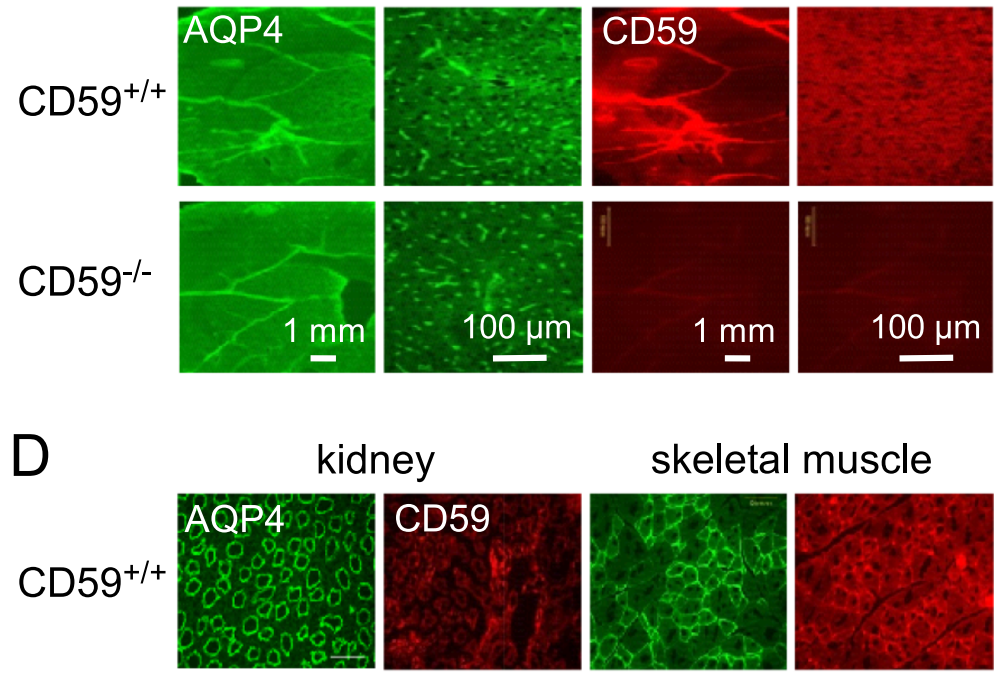

kidney

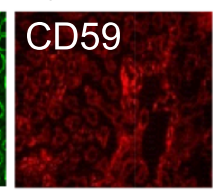

skeletal muscle

CD59-1-
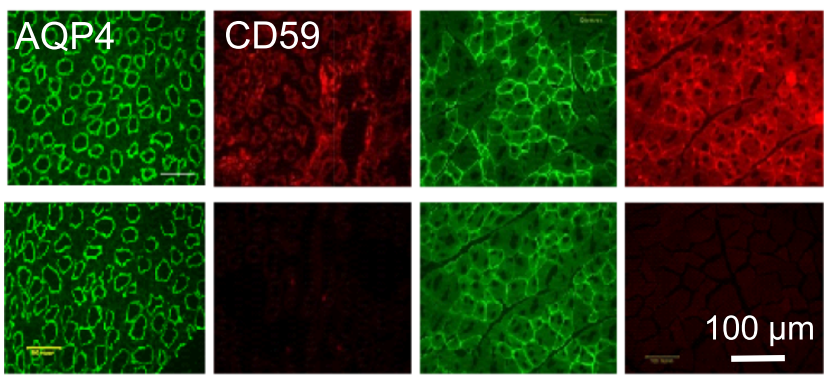

Fig. 2 AQP4 and CD59 expression in $\mathrm{CD} 59^{+/+}$and $\mathrm{CD} 59^{-/-}$rats. Immunofluorescence shown in cross-section and longitudinal section of spinal cord (a), optic nerves (b), coronal sections of brain (c), and kidney inner medulla and skeletal muscle sarcolemma (d). Representative of two mice per genotype

Immunofluorescence of spinal cord on day 3 showed marked loss of AQP4 and GFAP in cervical spinal cord of AQP4-IgG-treated CD59 $9^{-/-}$rats, with patchy and variable loss in thoracic and lumbar spinal cord (Fig. 5c, d). Minimal loss of AQP4 and GFAP was seen in treated CD $59^{+/+}$ rats. C5b-9 and Iba-1 immunofluorescence was seen in cervical and thoracic spinal cord in AQP4-IgG-treated
$\mathrm{CD} 59^{-/-}$rats (Fig. 5e). To investigate whether the location-dependent pathology in spinal cord is related to AQP4-IgG access and deposition, tissues from rats receiving $15 \mu \mathrm{g}$ AQP4-IgG were harvested at $2 \mathrm{~h}$ and immunostained with an anti-human secondary antibody. Figure $5 \mathrm{f}$ shows detectable human IgG in cervical > thoracic spinal cord, with little seen in lumbar spinal cord. 

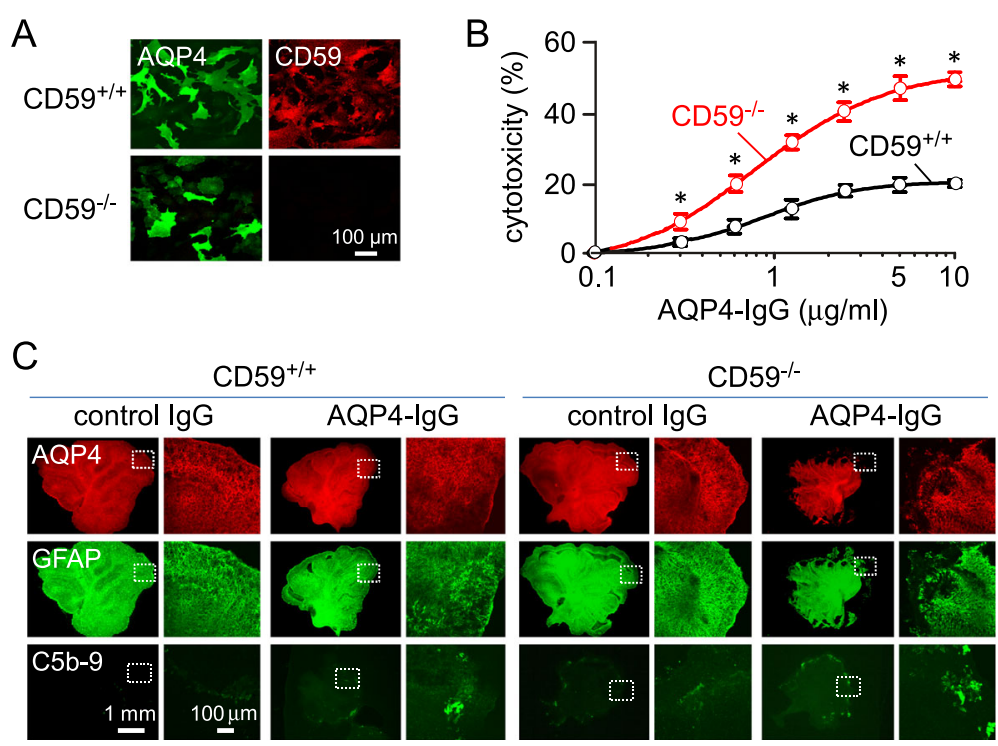

Fig. 3 Complement-mediated injury in $\mathrm{CD}_{5} 9^{+/+}$and $\mathrm{CD}_{5} 9^{-/-}$astrocyte cultures and cerebellar slices. a. AQP4 and CD59 immunofluorescence in primary astrocyte cultures from neonatal $\mathrm{CD} 59^{+/+}$and $\mathrm{CD}_{5} 9^{-/-}$rats. b. Complement-dependent cytotoxicity in astrocyte cultures following 2-h incubation with $5 \%$ human complement and indicated concentrations of AQP4-lgG (mean \pm S.E.M., $n=4$, * $P<0.01$ ). c. AQP4, GFAP and C5b-9 immunofluorescence in cerebellar slice cultures from $\mathrm{CD}_{5} 9^{+/+}$and $\mathrm{CD}_{59^{-/}}$rats at 1 day after incubation with $5 \mu \mathrm{g} / \mathrm{ml} \mathrm{AQP4-lgG} \mathrm{(or} \mathrm{control-lgG)} \mathrm{and} \mathrm{5 \%} \mathrm{human} \mathrm{complement.}$ Fluorescence micrographs shown as low and high (boxed region) magnifications. Representative of 3 sets of slice culture studies

Immunofluorescence of optic nerves showed patchy and variable loss of AQP4 and GFAP (results from 3 rats per genotype shown in Fig. 6a); C5b-9 and Iba-1 immunofluorescence were consistently greater in the AQP4-IgG treated $\mathrm{CD} 59^{-/-}$rats than the $\mathrm{CD} 59^{+/+}$rats. Brain sections showed patchy loss of AQP4 and GFAP, mainly at the brain surface and in periventricular matter, along with C5b-9 and Iba-1 immunofluorescence (Fig. 6b). AQP4-IgG (human IgG immunofluorescence) at $2 \mathrm{~h}$ after injection was not detectable in optic nerves, but seen in a patchy distribution in brain cortex and periventricular matter (Fig. 6c).

\section{Discussion}

Our study supports the central involvement of CD59 in modulating complement-mediated injury in AQP4-IgG seropositive NMO. CD59 is expressed in CNS tissues affected in NMO and may play a protective role to contain local, subclinical injury initiated by minor exposures to AQP4-IgG. CD59 ${ }^{-/-}$rats were highly sensitive to passive transfer of AQP4-IgG by intracerebral and intracisternal routes, without the need for added components such as complement or pro-inflammatory factors. Though astrocytes may also express other complement regulator proteins such as CD55, the marked effect of CD59 gene deletion suggests that CD59 is the major complement regulator in rat brain. As an important complement regulator in astrocytes, drugs that enhance astrocytic CD59 expression, perhaps identifiable by compound screens, may be beneficial in NMO, and conversely, reduced astrocytic CD59 expression or subcellular colocalization with AQP4 might trigger NMO exacerbations and worsen disease severity.

Animal models of NMO have been useful in characterizing NMO pathogenesis mechanisms and for testing potential NMO therapeutics. Mouse models have been developed involving passive transfer of AQP4-IgG together with human complement by direct injections into the brain [28] or spinal fluid [3,35] to produce NMOlike pathology in brain, spinal cord and optic nerve. As mentioned in the Introduction, a fundamental limitation of mice to study NMO is their lack of an effective classical complement activation pathway [5, 25]. Early rat models involved administration of AQP4-IgG following induction of experimental autoimmune encephalomyelitis (EAE) [4]; however, the pathogenic mechanism in EAE - myelin targeting by $\mathrm{T}$ cells - is very different from the humoral immune response in NMO, making it difficult to reach conclusions about NMO pathogenesis mechanisms. We found that intracerebral injection of AQP4-IgG produced robust NMO-like pathology in rat brain [1], and that while systemic administration of AQP4-IgG alone did not produce disease, NMO-like brain pathology was seen following a small needle stab in seropositive rats [2], which presumably allowed circulating AQP4-IgG leakage into brain parenchyma to access astrocytes, and perhaps produce a local inflammatory response. Creation of NMO spinal cord or optic nerve pathology in rats has been challenging. One study involving continuous AQP4-IgG infusion using intrathecal catheters 


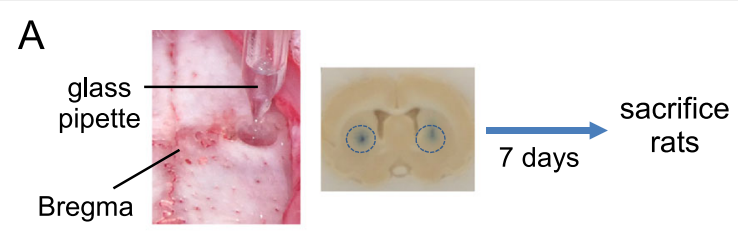

B

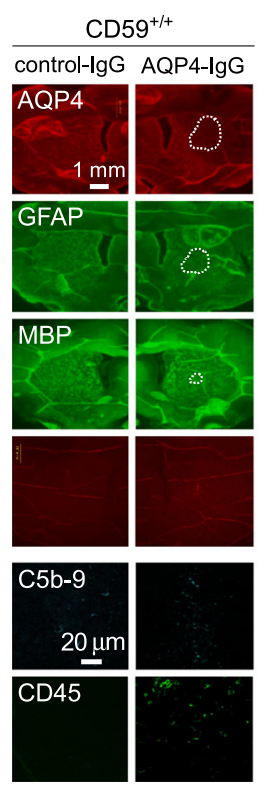

$\mathrm{CD} 59^{-/-}$

control-lgG $A Q P 4-I g G$
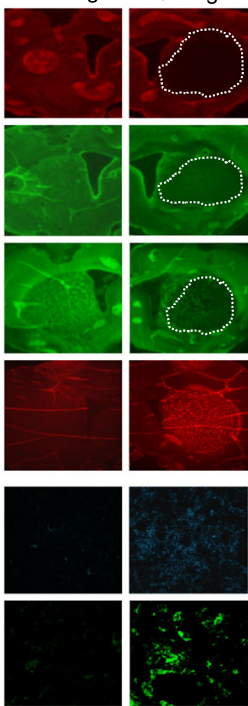

C

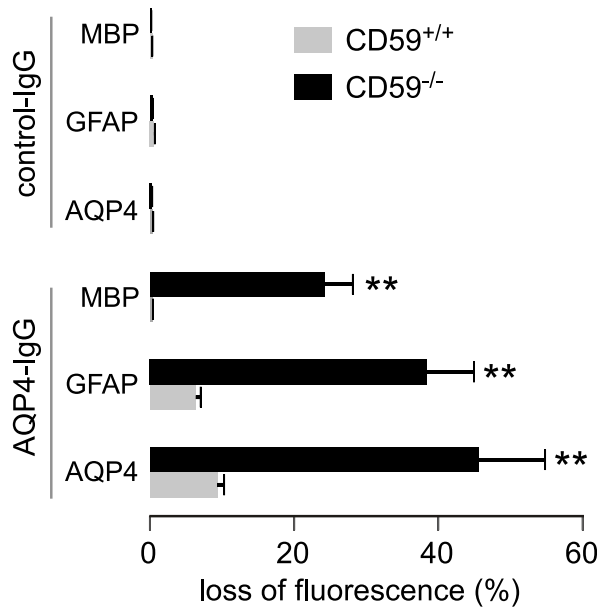

Fig. 4 Increased NMO pathology in $\mathrm{CD}^{-1-} 9^{-1}$ rats following intracerebral injection of AQP4-lgG. a. Intracerebral injection model showing stereotaxic microneedle injection of AQP4-lgG (or control lgG). b. Immunofluorescence of indicated markers in rat brain at 7 days after AQP4-lgG (or control lgG) injection. Lesion areas indicated by white dotted boundary. c. AQP4, GFAP and MPB immunodeficient areas normalized to hemisphere areas (mean \pm S.E.M., 6 rats per genotype, $\left.{ }^{* *} P<0.01\right)$

showed reversible AQP4 loss in spinal cord but without inflammation or demyelination [9], and a similar more recent study reported AQP4 loss in spinal cord and optic nerves, as well as mildly reduction in myelin in spinal cord [17]. The marked amplification of NMO pathology by knockout of CD59 in rats produced astrocytopathy as well as inflammation and deposition of activated complement.

$\mathrm{CD} 59^{-/-}$rats did not manifest overt phenotypes, except for mild reticulocytosis and reduced hemoglobin, which is likely due to low-grade hemolysis as seen in humans lacking CD59 [31] rather than a possible offtarget effect in genome editing that can occur using CRISPR methods. The active classical complement system in rats, which has similar activity to that in human $[5,33]$, is presumably the reason for the low basal hemolytic activity. As such, $\mathrm{CD} 59^{-/-}$rats may be useful to model complement-initiated diseases in various neurodegenerative, hematological, renal and skeletal muscle diseases [6, 11, 31]. Although the mechanism of high morbidity in $\mathrm{CD}_{59^{-/-}}$rats receiving cobra venom factor was not established here, there appeared to be hemolysis and organ injury, which is likely due to complement activation and consumption by cobra venom factor, which is the mechanism of its complement depletion action [32, 33]. With regard to NMO, the amplified response of $\mathrm{CD} 59^{-1-}$ rats to AQP4-IgG may be useful in testing drugs that target distinct steps in the AQP4-IgG/ complement injury pathway, as well as in investigating outstanding questions in NMO pathogenesis mechanisms such as the role of sensitized $\mathrm{T}$ cells and the explanation for the absence of significant pathology in peripheral AQP4-expressing tissues despite their sustained direct exposure to serum AQP4-IgG.

The marked NMO pathology seen in $\mathrm{CD} 59^{-/-}$rats following AQP4-IgG administration contrasts with the conclusions of Saadoun and Papadopoulos [27], who concluded that complement inhibitors, including CD59, are not protective against complement injury in CNS tissues. Their findings were based on immunofluorescence in mouse brain in which CD59 expression was seen on astrocytes, but not at AQP4-rich foot-processes abutting microvessels. Detection sensitivity rather than species differences may account for the disparate conclusions, as we previously showed marked $\mathrm{NMO}$ pathology in $\mathrm{CD} 59^{-/-}$mice following intracerebral or 

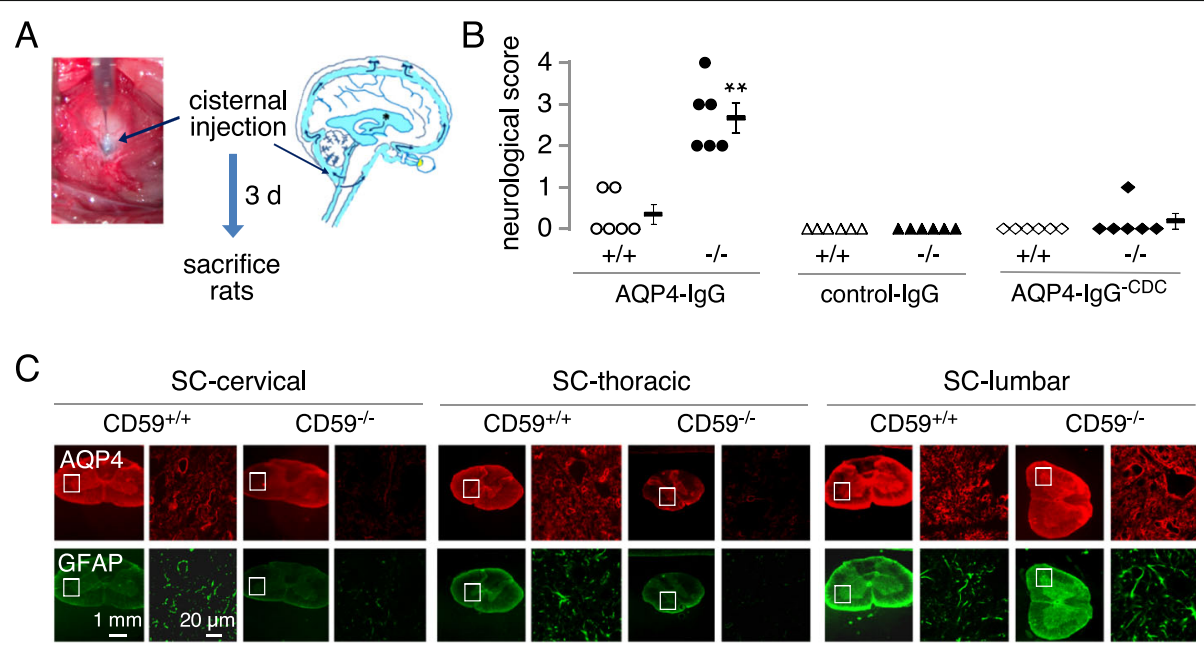

D

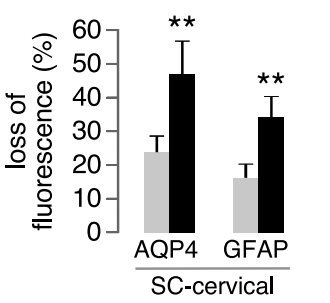

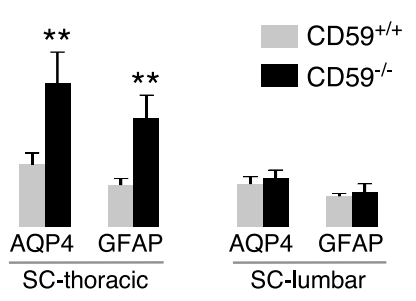

$\mathrm{E}$

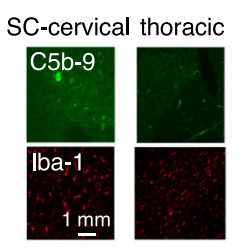

$\mathrm{F}$

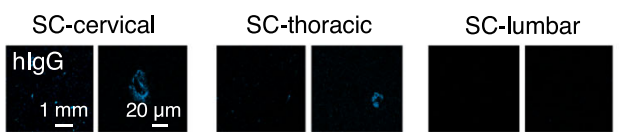

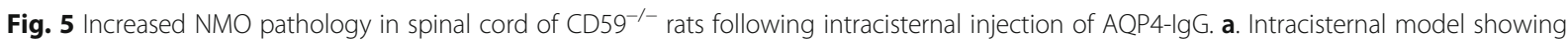
microneedle injection of AQP4-lgG (or control lgG). b. Neurological scores at day 3 after AQP4-lgG, control lgG, or engineered AQP4-lgG lacking complement effector function (AQP4-IgG ${ }^{-\mathrm{CDC}}$ ). Each symbol is data from a separate rat $(n=6)$, with mean \pm S.E.M. shown $(* * P<0.01)$. c. Immunofluorescence of indicated markers in cervical, thoracic and lumbar spinal cord at 3 days after AQP4-lgG injection. d. Loss of AQP4 and GFAP immunofluorescence normalized to whole section area of spinal cord (mean \pm S.E.M., 6 rats per genotype, ${ }^{* *} P<0.01$ ). e. C5b-9 and Iba-1 immunofluorescence in cervical and thoracic spinal cord at 3 days after AQP4-lgG injection. f. AQP4-lgG distribution at $2 \mathrm{~h}$ after intracisternal injection visualized with an anti-human secondary antibody

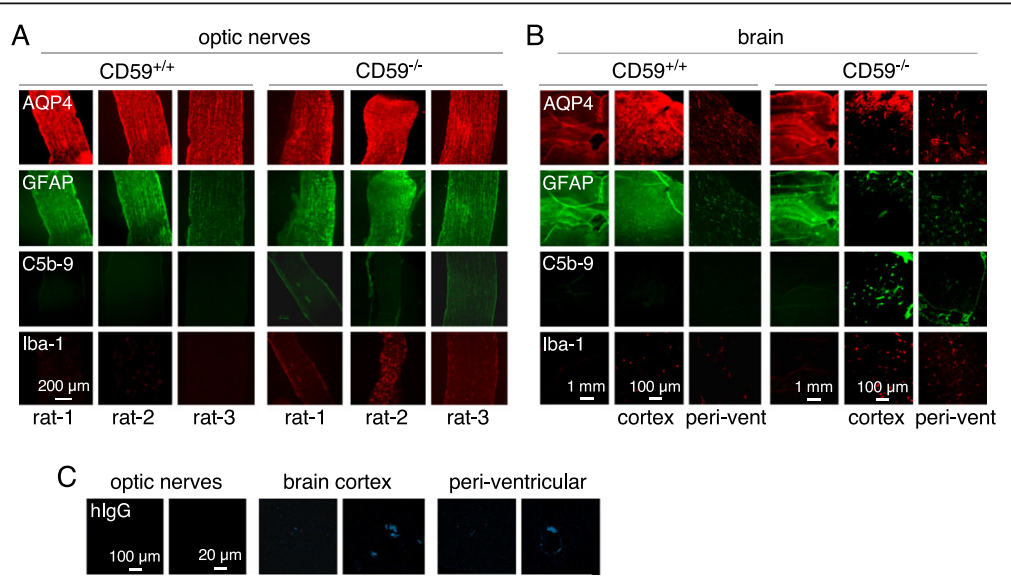

Fig. $6 \mathrm{NMO}$ pathology in optic nerves and brain of $\mathrm{CD}_{5} 9^{-/-}$rats following intracisternal injection of AQP4-lgG. a. Immunofluorescence of indicated markers in optic nerves at 3 days after AQP4-IgG injection. Data shown for 3 rats per genotype. $\mathbf{b}$. Immunofluorescence of indicated markers near the brain surface ('cortex') and around ventricles ('peri-vent') at 3 days after AQP4-lgG injection. c. Distribution of AQP4-lgG at $2 \mathrm{~h}$ after intracisternal injection visualized with an anti-human secondary antibody 
lumbosacral administration of AQP4-IgG with human complement [38]. Our recent development of super-resolution microscopy methods to image AQP4 on astrocytes in fixed CNS tissues [29] may overcome the limited resolution and sensitivity of conventional fluorescence microscopy to detect CD59 in subcellular regions of astrocytes. Saadoun and Papadopoulos [27] also speculated that the absence of significant NMO disease in peripheral AQP4-expressing tissues such as skeletal muscle and kidney was a consequence of CD59 and AQP4 coexpression, which should be amenable to testing using $\mathrm{CD} 59^{-/-}$rats.

\section{Conclusion}

In conclusion, our results implicate CD59 as an important regulator in NMO pathogenesis and potentially a new drug target with a novel mechanism of action to reduce complement-mediated astrocyte damage, a key initiating event in NMO. Prevention of complement-mediated astrocyte damage by altering astrocyte susceptibility to complement may have a more favorable side-effect profile than by general complement inhibition.

\section{Acknowledgments}

This work was supported by grants EY13574, EB00415, DK35124, and DK72517 from the National Institutes of Health, and a grant from the Guthy-Jackson Charitable Foundation. We thank Dr. Jeffrey Bennett (Univ. Colorado Denver, Aurora, $\mathrm{CO}$ ) for providing recombinant monoclonal NMO antibodies and TaO Su (UCSF) for help in astrocyte and slice culture studies.

\section{Authors' contributions}

$X Y$ carried out experiments and analyses. XY and ASV designed studies and wrote the manuscript. Both authors read and approved the final manuscript.

\section{Competing interests}

The authors declare that they have no competing interests.

Received: 29 December 2016 Accepted: 3 February 2017 Published online: 17 February 2017

\section{References}

1. Asavapanumas N, Ratelade J, Verkman AS (2014) Unique neuromyelitis optica pathology produced in naive rats by intracerebral administration of NMO-lgG. Acta Neuropathol 127:539-551

2. Asavapanumas N, Verkman AS (2014) Neuromyelitis optica pathology in rats following intraperitoneal injection of $\mathrm{NMO}-\mathrm{lgG}$ and intracerebral needle injury. Acta Neuropathol Commun 2:48

3. Asgari N, Khorooshi R, Lillevang ST, Owens T (2013) Complementdependent pathogenicity of brain-specific antibodies in cerebrospinal fluid. J Neuroimmunol 254:76-82

4. Bennett JL, Lam C, Kalluri SR, Saikali P, Bautista K, Dupree C, Glogowska M, Case D, Antel JP, Owens GP, Gilden D, Nessler S, Stadelmann C, Hemmer B (2009) Intrathecal pathogenic anti-aquaporin-4 antibodies in early neuromyelitis optica. Ann Neurol 66:617-629

5. Bergman I, Basse PH, Barmada MA, Griffin JA, Cheung NK (2000) Comparison of in vitro antibody-targeted cytotoxicity using mouse, rat and human effectors. Cancer Immunol Immunother 49:259-266

6. Brodsky RA (2015) Complement in hemolytic anemia. Blood 126:2459-2465

7. Felix CM, Levin MH, Verkman AS (2016) Complement-independent retinal pathology produced by intravitreal injection of neuromyelitis optica immunoglobulin G. J Neuroinflammation 13:275

8. Fujihara K (2011) Neuromyelitis optica and astrocytic damage in its pathogenesis. Journal of neurological sciences 306:183-187

9. Geis C, Ritter C, Ruschil C, Weishaupt A, Grunewald B, Stoll G, Holmoy T, Misu T, Fujihara K, Hemmer B, Stadelmann C, Bennett JL, Sommer C, Toyka KV (2015) The intrinsic pathogenic role of autoantibodies to aquaporin 4 mediating spinal cord disease in a rat passive-transfer model. Exp Neurol 265:8-21

10. Hengstman GJ, Wesseling P, Frenken CW, Jongen PJ (2007) Neuromyelitis optica with clinical and histopathological involvement of the brain. Mult Scler 13:679-682

11. Holers VM, Thurman JM (2004) The alternative pathway of complement in disease:opportunities for therapeutic targeting. Mol Immunol 41:147-152

12. Iwamoto N, Kawaguchi T, Nagakura S, Hidaka M, Horikawa K, Kagimoto T, Takatsuki K, Nakakuma H (1995) Markedly high population of affected reticulocytes negative for decay-accelerating factor and CD59 in paroxysmal nocturnal hemoglobinuria. Blood 85:2228-2232

13. Jarius S, Wildemann B (2010) AQP4 antibodies in neuromyelitis optica: diagnostic and pathogenetic relevance. Nat Rev Neurol 6:383-392

14. Lennon VA, Kryzer TJ, Pittock SJ, Verkman AS, Hinson SR (2005) lgG marker of optic-spinal multiple sclerosis binds to the aquaporin-4 water channel. J Exp Med 202:473-477

15. Li L, Zhang H, Varrin-Doyer M, Zamvil SS, Verkman AS (2011) Proinflammatory role of aquaporin-4 in autoimmune neuroinflammation. FASEB J 25:1556-1566

16. Lucchinetti CF, Mandler RN, McGavern D, Bruck W, Gleich G, Ransohoff RM, Trebst C, Weinshenker B, Wingerchuk D, Parisi JE, Lassmann H (2002) A role for humoral mechanisms in the pathogenesis of Devic's neuromyelitis optica. Brain 125:1450-1461

17. Marignier R, Ruiz A, Cavagna S, Nicole A, Watrin C, Touret M, Parrot S, Malleret G, Peyron C, Benetollo C, Auvergnon N, Vukusic S, Giraudon P (2016) Neuromyelitis optica study model based on chronic infusion of autoantibodies in rat cerebrospinal fluid. J Neuroinflammation 13:111

18. Misu T, Fujihara K, Kakita A, Konno H, Nakamura M, Watanabe S, Takahashi T, Nakashima I, Takahashi H, Itoyama Y (2007) Loss of aquaporin 4 in lesions of neuromyelitis optica: distinction from multiple sclerosis. Brain 130:1224-1234

19. Papadopoulos MC, Verkman AS (2012) Aquaporin 4 and neuromyelitis optica. The Lancet Neurology 11:535-544

20. Perez-Nievas BG, Garcia-Bueno B, Madrigal JL, Leza JC (2010) Chronic immobilisation stress ameliorates clinical score and neuroinflammation in a MOG-induced EAE in Dark Agouti rats: mechanisms implicated. J Neuroinflammation 7:60

21. Pittock SJ, Lennon VA, McKeon A, Mandrekar J, Weinshenker BG, Lucchinetti CF, O'Toole O, Wingerchuk DM (2013) Eculizumab in AQP4-lgG-positive relapsing neuromyelitis optica spectrum disorders: an open-label pilot study. The Lancet Neurology 12:554-562

22. Pohl M, Fischer MT, Mader S, Schanda K, Kitic M, Sharma R, Wimmer I, Misu T, Fujihara K, Reindl M, Lassmann H, Bradl M (2011) Pathogenic T cell responses against aquaporin 4. Acta Neuropathol 122:21-34

23. Qin X, Krumrei N, Grubissich L, Dobarro M, Aktas H, Perez G, Halperin JA (2003) Deficiency of the mouse complement regulatory protein $\mathrm{mCd} 59 \mathrm{~b}$ results in spontaneous hemolytic anemia with platelet activation and progressive male infertility. Immunity 18:217-227

24. Ratelade J, Asavapanumas N, Ritchie AM, Wemlinger S, Bennett JL, Verkman AS (2013) Involvement of antibody-dependent cell-mediated cytotoxicity in inflammatory demyelination in a mouse model of neuromyelitis optica. Acta Neuropathol 126:699-709

25. Ratelade J, Verkman AS (2014) Inhibitor(s) of the classical complement pathway in mouse serum limit the utility of mice as experimental models of neuromyelitis optica. Mol Immunol 62:104-113

26. Roemer SF, Parisi JE, Lennon VA, Benarroch EE, Lassmann H, Bruck W, Mandler RN, Weinshenker BG, Pittock SJ, Wingerchuk DM, Lucchinetti CF (2007) Pattern-specific loss of aquaporin-4 immunoreactivity distinguishes neuromyelitis optica from multiple sclerosis. Brain 130:1194-1205

27. Saadoun S, Papadopoulos MC (2015) Role of membrane complement regulators in neuromyelitis optica. Mult Scler 21:1644-1654

28. Saadoun S, Waters $P$, Bell BA, Vincent A, Verkman AS, Papadopoulos MC (2010) Intra-cerebral injection of neuromyelitis optica immunoglobulin $G$ and human complement produces neuromyelitis optica lesions in mice. Brain 133:349-361

29. Smith AJ, Verkman AS (2015) Superresolution imaging of aquaporin-4 cluster size in antibody-stained paraffin brain sections. Biophys J 109:2511-2522

30. Tradtrantip L, Ratelade J, Zhang H, Verkman AS (2013) Enzymatic deglycosylation converts pathogenic neuromyelitis optica anti-aquaporin-4 immunoglobulin G into therapeutic antibody. Ann Neurol 73:77-85

31. Unsworth DJ (2008) Complement deficiency and disease. J Clin Pathol 61: 1013-1017 
32. Van den Berg CW, Aerts PC, Van Dijk H (1991) In vivo anti-complementary activities of the cobra venom factors from Naja naja and Naja haje. J Immunol Methods 136:287-294

33. Vogel CW, Fritzinger DC (2010) Cobra venom factor: Structure, function, and humanization for therapeutic complement depletion. Toxicon 56:1198-1222

34. Yao X, Su T, Verkman AS (2016) Clobetasol promotes remyelination in a mouse model of neuromyelitis optica. Acta Neuropathol Commun 4:42

35. Zeka B, Hastermann M, Hochmeister S, Kogl N, Kaufmann N, Schanda K, Mader S, Misu T, Rommer P, Fujihara K, Illes Z, Leutmezer F, Sato DK, Nakashima I, Reindl M, Lassmann H, Bradl M (2015) Highly encephalitogenic aquaporin 4-specific T cells and NMO-lgG jointly orchestrate lesion location and tissue damage in the CNS. Acta Neuropathol 130:783-798

36. Zeka B, Hastermann M, Kaufmann N, Schanda K, Pende M, Misu T, Rommer P, Fujihara K, Nakashima I, Dahle C, Leutmezer F, Reindl M, Lassmann H, Bradl M (2016) Aquaporin 4-specific T cells and NMO-lgG cause primary retinal damage in experimental NMO/SD. Acta Neuropathol Commun 4:82

37. Zhang $H$, Verkman AS (2013) Eosinophil pathogenicity mechanisms and therapeutics in neuromyelitis optica. J Clin Invest 123:2306-2316

38. Zhang H, Verkman AS (2014) Longitudinally extensive NMO spinal cord pathology produced by passive transfer of NMO-lgG in mice lacking complement inhibitor CD59. J Autoimmun 53:67-77

\section{Submit your next manuscript to BioMed Central} and we will help you at every step:

- We accept pre-submission inquiries

- Our selector tool helps you to find the most relevant journal

- We provide round the clock customer support

- Convenient online submission

- Thorough peer review

- Inclusion in PubMed and all major indexing services

- Maximum visibility for your research

Submit your manuscript at www.biomedcentral.com/submit 